\title{
Análise de crescimento e partição de assimilados em plantas de tomateiro cv. Micro-Tom submetidas ao nitrogênio e piraclostrobina
}

\author{
Growth analysis partitioning of assimilate in tomato plants $\mathrm{cv}$. \\ Micro-Tom submitted to nitrogen and pyraclostrobin
}

\author{
Emanuela Garbin Martinazzo ${ }^{1 *}$; Anelise Tessari Perboni²; Douglas Antonio Posso ${ }^{3}$; \\ Tiago Zanatta Aumonde ${ }^{4}$; Marcos Antonio Bacarin ${ }^{5}$
}

\begin{abstract}
Resumo
Este trabalho objetivou analisar comparativamente o crescimento e a partição de assimilados em plantas de tomateiro cv. Micro-Tom submetidas ao nitrogênio e piraclostrobina. Esta substância favorece o desenvolvimento de cloroplastos e a síntese de clorofila. Plantas de tomateiro foram submetidas aos tratamentos: T1, solução nutritiva completa sem piraclostrobina; T2, solução nutritiva completa + piraclostrobina; $\mathrm{T} 3$, solução nutritiva $1 / 2$ força de $\mathrm{N}$ sem piraclostrobina e $\mathrm{T} 4$, solução nutritiva $1 / 2$ força de $\mathrm{N}+$ piraclostrobina. As plantas foram coletadas a intervalos regulares de sete dias após o transplante ao longo do ciclo de cultivo, sendo determinados a massa seca e a área foliar. A partir dos dados primários, foi aplicada a análise de crescimento, sendo calculados a massa seca total $\left(\mathrm{W}_{\mathrm{t}}\right)$, as taxas instantâneas de produção de matéria seca $\left(\mathrm{C}_{\mathrm{t}}\right)$, crescimento relativo $\left(\mathrm{R}_{\mathrm{w}}\right)$ e assimilatória líquida $\left(\mathrm{E}_{\mathrm{a}}\right)$, a área foliar $\left(\mathrm{A}_{\mathrm{f}}\right)$, as taxas de produção $\left(\mathrm{C}_{\mathrm{a}}\right)$ e de crescimento relativo de área foliar $\left(\mathrm{R}_{\mathrm{a}}\right)$, as razões de área foliar $\left(\mathrm{F}_{\mathrm{a}}\right)$ e de massa foliar $\left(\mathrm{F}_{\mathrm{w}}\right)$, a área foliar específica $\left(\mathrm{S}_{\mathrm{a}}\right)$, a partição de matéria seca entre órgãos e o número $\left(\mathrm{N}_{\mathrm{fr}}\right)$ e massa fresca de frutos $\left(\mathrm{W}_{\mathrm{fr}}\right)$. Plantas de $\mathrm{T} 1$ apresentaram maior $\mathrm{W}_{\mathrm{t}}, \mathrm{C}_{\mathrm{t}} \mathrm{e} \mathrm{W}_{\mathrm{fr}}$ em relação àquelas dos demais tratamentos. Entretanto, plantas de $\mathrm{T} 2$ apresentaram similar $\mathrm{N}_{\mathrm{fr}}$ à plantas de $\mathrm{T} 1$, sendo superiores às demais. Além disso, alocaram em relação à matéria seca total e ao final do ciclo, maior porcentagem de matéria seca nos frutos comparativamente a plantas de T3 e T4. A associação entre nitrogênio e piraclostrobina altera o crescimento e a partição de assimilados em plantas de tomateiro cv. MicroTom, sendo que àquelas submetidas à $1 / 2$ dose de nitrogênio apresentam maior massa seca total e menor porcentagem final de matéria seca total em frutos, comparativamente, àquelas submetidas à associação $1 / 2$ dose de nitrogênio e a piraclostrobina.
\end{abstract}

Palavras-chave: Lycopersicon esculentum, estrobilurina, bioativador, massa seca, área foliar

\begin{abstract}
This work aimed at comparing the growth and partitioning of assimilate in tomato plants cv. Micro-Tom subjected to nitrogen and pyraclostrobin. This substance favors the development of chloroplasts and the synthesis of chlorophyll. Tomato plants were submitted to the treatments: T1, complete nutrient solution without pyraclostrobin, T2, complete nutrient solution + pyraclostrobin, T3, 1/2 strength nutrient solution
\end{abstract}

1 Prof ${ }^{\mathrm{a}} \mathrm{Dr}^{\mathrm{a}}$, Instituto de Ciências Biológicas, Universidade Federal do Rio Grande, FURG, Rio Grande, RS. E-mail: emartinazzo@ furg.br

2 Prof $^{a}$ Dra $^{\mathrm{a}}$, Instituto de Ciências e Tecnologia das Águas, Universidade Federal do Oeste do Pará, UFOPA, Santarém, PA. E-mail: aneperboni@yahoo.com.br

3 Discente, Bolsista de Iniciação Científica PIBIC CNPq, Instituto de Biologia, Universidade Federal de Pelotas, UFPel, Pelotas, RS. E-mail: douglasposso@hotmail.com

4 Prof. Dr., Faculdade de Agronomia Eliseu Maciel, Dept ${ }^{\circ}$ de Fitotecnia, UFPel, Pelotas, RS. E-mail: tiago.aumonde@gmail.com

5 Prof. Dr., Instituto de Biologia, UFPel, Pelotas, RS. E-mail: bacarin@ufpel.edu.br

* Autor para correspondência 
without $\mathrm{N}$ pyraclostrobin and T4, 1/2 strength nutrient solution $\mathrm{N}+$ pyraclostrobin. Plants were collected at regular intervals of seven days after transplantation throughout the crop cycle, with dry mass and leaf area being determined. From the primary data, growth analysis was carried out to calculate total dry matter $\left(\mathrm{W}_{\mathrm{t}}\right)$, the instantaneous rates of dry matter production $\left(\mathrm{C}_{\mathrm{t}}\right)$, relative growth $\left(\mathrm{R}_{\mathrm{w}}\right)$ e net assimilation $\left(\mathrm{E}_{\mathrm{a}}\right)$, leaf area $\left(\mathrm{A}_{\mathrm{f}}\right)$, production rates $\left(\mathrm{C}_{\mathrm{a}}\right)$ and relative growth of leaf area index $\left(\mathrm{R}_{\mathrm{a}}\right)$ and leaf weight $\left(\mathrm{F}_{\mathrm{w}}\right)$ specific leaf area $\left(\mathrm{S}_{\mathrm{a}}\right)$ the dry matter partitioning between organs and number $\left(\mathrm{N}_{\mathrm{fr}}\right)$ and fresh fruit weight $\left(\mathrm{W}_{\mathrm{fr}}\right)$. Plants of $\mathrm{T} 1$ showed higher $\mathrm{W}_{\mathrm{t}}, \mathrm{C}_{\mathrm{t}}$ and $\mathrm{W}_{\mathrm{fr}}$ compared to those of other treatments. However, the $\mathrm{T} 2$ plants exhibited similar Nfr to T1 plants, being superior to others. Also allocated on the total dry matter and at the end of the cycle, a higher percentage of dry matter in the seafood compared to T3 and T4 plants. Also they allocated relative to the total dry matter and at the end of the cycle, a higher percentage in fruits of plants to $\mathrm{T} 3$ and $\mathrm{T} 4$. The association between nitrogen and pyraclostrobin changes the growth and assimilated partition on tomato plants $\mathrm{cv}$. Micro - Tom, and those submitted to $1 / 2$ dose of nitrogen have a higher total dry matter and less final percentage of total dry matter in fruits, comparatively to those submitted to the association $1 / 2$ dose of nitrogen and pyraclostrobin.

Key words: Lycopersicon esculentum, strobilurin, bioactivator, dry mass, leaf area

\section{Introdução}

No contexto da agricultura moderna e com a evolução de técnicas de cultivo, compostos ou produtos químicos com efeito estimulante no crescimento e desenvolvimento de espécies vegetais tem merecido atenção especial, principalmente para culturas cujo produto final seja dotado de elevado valor agregado. Neste sentido, reguladores de crescimento constituem-se de compostos orgânicos, que não sejam macro ou micronutrientes, com capacidade de alterar a fisiologia, o crescimento e o desenvolvimento vegetal. Entre os reguladores de crescimento estão o ácido abscísico, auxina, giberelina, etileno e citocinina (TAIZ; ZEIGER, 2013). Já, os bioestimulantes, são compostos pela combinação de reguladores de crescimento com substâncias de natureza química diferente, enquanto, substâncias orgânicas complexas com a capacidade de alterar o crescimento, a expressão gênica, de proteínas de membrana e a atividade enzimática são denominadas bioativadores (CASTRO et al., 2007).

As estrobilurinas pertencem à classe de fungicidas de amplo espectro de ação que interrompe a cadeia de transporte de elétrons da mitocôndria, diminuindo a produção de trifosfato de adenosina, impedindo a germinação de esporos e, consequentemente, reduzindo o crescimento micelial de fungos (VENANCIO et al., 2003). Além do controle de doenças, provoca alterações na fisiologia do vegetal tendo em vista que, plantas não infectadas quando tratadas com fungicidas a base de estrobilurinas tornam-se mais sadias, resultado do efeito verdejante ("greening") (BARTLETT et al., 2002; KÖHLER et al., 2002).

Estudos conduzidos com estrobilurinas demonstram decréscimo na produção de etileno (GROSSMANN; RETZLAFF, 1997) que, por sua vez, retarda a degradação de citocininas, favorecendo o desenvolvimento de cloroplastos e a síntese de clorofila. Este modo de ação pode alterar características morfológicas da planta aumentando sua capacidade em manter a área foliar verde, maximizando a produtividade da cultura (BARTLETT et al., 2002).

Além de mudanças morfológicas resultantes do tratamento com estrobilurinas, uma variedade de processos fisiológicos dos vegetais podem ser diretamente afetados, como melhor utilização do dióxido de carbono e maior acúmulo de carboidratos, diminuição da senescência foliar, aumento da atividade de enzimas antioxidantes e da redutase do nitrato (ZHANG et al., 2010), afetando a dinâmica do nitrogênio. Neste contexto, o tratamento com o fungicida pode afetar a absorção de nitrogênio disponível no solo, alterar o crescimento e a partição de assimilados ao longo do ciclo de desenvolvimento de espécies cultivadas, refletindo em incrementos de produtividade. 
A utilização de espécies modelo visando o estudo do comportamento vegetal frente à aplicação de agroquímicos ou agrotóxicos é importante ferramenta na avaliação do seu efeito sobre a fisiologia do crescimento e do desenvolvimento de plantas. A cultivar de tomateiro Micro-Tom (Lycopersicon esculentum) é de porte pequeno, crescimento rápido, ciclo médio de 70-90 dias, produz frutos e sementes viáveis, podendo ser cultivada em recipientes com reduzido volume de substrato (MARTÍ et al., 2006). Estes fatores possibilitam a rápida coleta de dados de crescimento e a caraterização do efeito de determinado produto sobre o desempenho vegetal ao longo do ciclo de cultivo, constituindo importante ferramenta no estudo da adaptação da planta sob diferentes condições de meio e manejo.

O presente trabalho teve como objetivo analisar comparativamente por meio da equação logística simples o crescimento e a partição de assimilados em plantas de tomateiro cv. Micro-Tom submetidas ao nitrogênio e piraclostrobina.

\section{Material e Métodos}

Sementes de tomateiro (Lycopersicon esculentum Mill) cv. Micro-Tom foram dispostas para germinar em caixas tipo "gerbox" sobre duas folhas de papel mata-borrão umedecidas com água destilada na proporção de 2,5 vezes a massa seca do papel seco e transferidas para câmara de germinação tipo BOD a temperatura de $25 \pm 2{ }^{\circ} \mathrm{C}$ e luz constante (BRASIL, 2009), mantida por quatro lâmpadas brancas fluorescentes de $25 \mathrm{~W}$, tipo luz do dia, onde permaneceram por dez dias. Após este período, as plântulas foram transplantadas para vasos de polietileno com volume de $500 \mathrm{~mL}$ de substrato, contendo areia lavada e uma planta por vaso.

As plantas foram cultivadas em casa de vegetação revestida com filme de polietileno de baixa densidade, situada na latitude $31^{\circ} 52^{\prime} \mathrm{S}$, longitude $52^{\circ} 21^{\prime} \mathrm{W}$ e altitude $13 \mathrm{~m}$, em área experimental do Departamento de Botânica, Universidade Federal de Pelotas, Capão do Leão, RS.
A irrigação foi efetuada por meio do sistema de microaspersão, com tempo de irrigação de dois minutos e intervalo de seis horas. Na primeira semana após o transplante das mudas, três vezes por semana, foi aplicada solução nutritiva de Hoagland e Arnon (1950).

Aos 21 dias após o transplante (DAT), as plantas foram submetidas a quatro tratamentos: $\mathrm{T} 1$ - solução nutritiva completa sem aplicação de piraclostrobina; T2 - solução nutritiva completa + aplicação piraclostrobina; T3 - solução nutritiva $1 / 2$ força de nitrogênio sem aplicação de piraclostrobina e T4, solução nutritiva $1 / 2$ força de nitrogênio + aplicação de piraclostrobina. A aplicação do princípio ativo piraclostrobina foi realizada aos 21; 35 e 49 DAT por meio do produto Comet $^{\circledR}$ na dose de $40 \mathrm{~mL}$ $100 \mathrm{~L}^{-1}$ de água utilizando o volume da calda de $1000 \mathrm{~L} \mathrm{ha}^{-1}$ (25\% i.a - piraclostrobina), conforme recomendação do fabricante.

Para a obtenção dos dados primários de crescimento de área foliar e massa da matéria seca, foram efetuadas coletas sucessivas a intervalos regulares de tempo de sete dias ao longo do ciclo de desenvolvimento das plantas. Em cada coleta, as plantas foram cortadas rente ao solo, separadas em órgãos (folhas, caule, flores e frutos) e acondicionadas em sacos de papel. As raízes foram lavadas sobre peneira de malha fina com auxílio de água corrente e, para a obtenção da matéria seca, o material foi transferido para estufa de ventilação forçada a temperatura de $65 \pm 2{ }^{\circ} \mathrm{C}$, até massa constante.

A área foliar das lâminas verdes $\left(\mathrm{A}_{\mathrm{f}}\right)$ foi determinada com medidor de área Li-Cor modelo LI-3100 e expressa em centímetros quadrados por planta $\left(\mathrm{cm}^{2}\right.$ planta $\left.^{-1}\right)$. Os dados primários de matéria seca total acumulada $\left(\mathrm{W}_{\mathrm{t}}\right)$ foram ajustados pela equação logística simples: $\mathbf{W}_{\mathrm{t}}=\mathbf{W}_{\mathrm{m}} /\left(\mathbf{1}+\mathbf{A e ^ { - \mathrm { Bt } } )}\right.$, sendo " $\mathbf{W}_{\mathbf{m}}$ " a estimativa assintótica do crescimento máximo, "A" e "B" constantes de ajustamento, "e" a base natural de logaritmo neperiano e " $\mathbf{t}$ " o tempo 
em dias após o transplante (RICHARDS, 1969); $\mathrm{e}$, os dados primários de área foliar $\left(\mathrm{A}_{\mathrm{f}}\right)$ ajustados por meio de polinômios ortogonais (RICHARDS, 1969). Os valores instantâneos da taxa de produção de matéria seca $\left(\mathrm{C}_{\mathrm{t}}\right)$ foram determinados por meio de derivadas das equações ajustadas da matéria seca total $\left(\mathrm{W}_{\mathrm{t}}\right.$ ) em relação ao tempo (RADFORD, 1967). Para a determinação dos valores instantâneos da taxa de crescimento relativo $\left(\mathrm{R}_{\mathrm{w}}\right)$ e taxa de crescimento relativo de área foliar $\left(R_{a}\right)$ empregadas as fórmulas: $R_{w}=1 / W_{t} \cdot d_{w} / d_{t}$ e $R_{a}=1 / A_{f} \cdot d A_{f} / d_{t}$ - Os valores instantâneos da taxa assimilatória líquida $\left(\mathrm{E}_{\mathrm{a}}\right)$, a razão de área foliar $\left(\mathrm{F}_{\mathrm{a}}\right)$, a razão de massa foliar $\left(\mathrm{F}_{\mathrm{w}}\right)$; a área foliar específica $\left(\mathrm{S}_{\mathrm{a}}\right)$ e a taxa de produção de área foliar $\left(\mathrm{C}_{\mathrm{o}}\right)$ foram estimados por meio das equações: $\mathbf{E}_{\mathrm{a}}=\mathbf{1} / \mathbf{A}_{\mathbf{f}} \cdot \mathbf{d}_{\mathbf{w}} / \mathbf{d}_{\mathrm{t}}$; $\mathrm{F}_{\mathrm{a}}={ }_{\mathrm{A}} / \mathrm{W}_{\mathrm{t}} ; \mathrm{F}_{\mathrm{w}}=\mathrm{W}_{\mathrm{f}} / \mathrm{W}_{\mathrm{t}} ; \mathrm{s}_{\mathrm{a}}={ }_{\mathrm{A}} / \mathrm{W}_{\mathrm{f}}$ e $\mathrm{C}_{\mathrm{t}}=\mathrm{dA}_{\mathrm{f}} / \mathrm{dt}$, conforme Radford (1967), o número de frutos $\left(\mathrm{N}_{\mathrm{fr}}\right)$ determinado a partir da contagem direta de frutos por planta e a massa fresca de frutos $\left(\mathrm{W}_{\mathrm{fr}}\right)$ aferida em balança de precisão. A partição de assimilados ao longo do desenvolvimento das plantas foi obtida pela transformação dos dados primários de alocação de massa seca de cada órgão para porcentagem.

Os dados relativos ao número de frutos e massa fresca de frutos foram submetidos à análise de variância e quando significativo, comparados pelo teste de Tukey a $5 \%$ de probabilidade. O delineamento experimental foi inteiramente casualizado e, em cada coleta, foram utilizadas quatro plantas escolhidas ao acaso, cada planta constituiu uma repetição, totalizando quatro repetições e onze coletas.

\section{Resultados e Discussão}

A matéria seca total das plantas $\left(\mathrm{W}_{\mathrm{t}}\right)$ ajustouse a tendência logística $\left(\mathrm{R}^{2}=0,99\right)$ em todos os tratamentos (Tabela 1 e Figura 1A). Indiferentemente ao tratamento, houve período de lento crescimento inicial das plantas até os 35 DAT seguido por fase de rápido crescimento até os 84 DAT. Ocorreu expressiva diferença na produção de matéria seca máxima, sendo maior em plantas submetidas a solução nutritiva completa sem aplicação de piraclostrobina (4,35 $\left.\mathrm{g} \mathrm{planta}^{-1}\right)$ e com aplicação de piraclostrobina $\left(3,49 \mathrm{~g}\right.$ planta $\left.^{-1}\right)$ comparativamente às plantas submetidas a solução nutritiva $1 / 2$ força de nitrogênio sem aplicação de piraclostrobina $(2,22 \mathrm{~g}$ planta $\left.^{-1}\right)$ e com aplicação de piraclostrobina $(2,09$ g planta $^{-1}$ ), que mantiveram similar produção de matéria seca total.

A taxa de produção de matéria seca $\left(\mathrm{C}_{\mathrm{t}}\right)$ foi baixa no início do ciclo de desenvolvimento das plantas e corrobora à diminuta produção de matéria seca total no referido período (Figura 1A e 1B). Houve, em todos os tratamentos, elevação na $\mathrm{C}_{\mathrm{t}}$ a partir dos 35 DAT sendo a máxima $C_{t}$ atingida aos $56,59,52$ e 52, para plantas dos tratamentos T1, T2, T3 e T4, respectivamente. É possível verificar a ocorrência de alteração temporal-quantitativa-negativa na taxa de produção de matéria seca em plantas submetidas à solução nutritiva completa e ausência de piraclostrobina (T1), pois houve atraso de 4 dias comparativamente àquelas submetidas a T3 e T4. Contudo, ocorreu precocidade de 3 dias para atingir a máxima $C_{t}$ em relação a $T 2$, evidenciando modificação temporal-quantitativa-positiva neste atributo de crescimento. 
Tabela 1: Equações ajustadas para matéria seca total e área foliar, para plantas de tomateiro cv. Micro-Tom submetidas ao nitrogênio e piraclostrobina.

\begin{tabular}{|c|c|c|c|c|}
\hline Tratamentos & Matéria seca total & $\mathbf{R}^{2}$ & Área foliar & $\mathbf{R}^{2}$ \\
\hline T1 & $4,43 / 1-255,2 e^{-0,0977 t}$ & 0,99 & $111,3+7,69 x-0,065 x^{\mathbf{2}}+6,13 \cdot 10^{-5} x^{\mathbf{3}}$ & 0,89 \\
\hline $\mathbf{T 2}$ & $3,58 / 1-240,5 e^{-0,093 \mathbf{2} t}$ & 0,99 & $69,3+4,87 x-0,035 x^{2}+3,3 \cdot 10^{-5} x^{3}$ & 0,90 \\
\hline T3 & $2,26 / 1-121,0 e^{-0,0889 t}$ & 0,99 & $64,1+4,59 x-0,036 x^{2}+2,69.10^{-5} x^{3}$ & 0,90 \\
\hline T4 & $2,04 / 1-243,3 e^{-0,1031 t}$ & 0,99 & $52,3+3,87 x-0,030 x^{2}+1,02 \cdot 10^{-5} x^{3}$ & 0,89 \\
\hline
\end{tabular}

Fonte: Elaboração dos autores.

As curvas da taxa de crescimento relativo $\left(\mathrm{R}_{\mathrm{w}}\right)$ demonstraram que plantas de tomateiro cv MicroTom apresentaram comportamento típico, sendo elevada no início com posterior queda ao longo do ciclo (Figura 1C). Foi observada variação no valor máximo de $\mathrm{R}_{\mathrm{w}}$, sendo de $0,105 \mathrm{~g}_{\text {planta }}{ }^{-1}$ dia $^{-1}$ obtido para plantas do tratamento com solução nutritiva $1 / 2$ força de nitrogênio + piraclostrobina (T4); 0,100 g planta ${ }^{-1}$ dia $^{-1}$ para plantas do tratamento com solução nutritiva completa sem piraclostrobina (T1); 0,091 g planta ${ }^{-1}$ dia $^{-1}$ para plantas submetidas a solução nutritiva completa + piraclostrobina (T2) e 0,089 g planta $^{-1}$ dia $^{-1}$ atingido por plantas submetidas a solução nutritiva $1 / 2$ força de nitrogênio sem piraclostrobina (T3). Assim, é possível evidenciar que a aplicação de piraclostrobina proporcionou maior eficiência na alocação de matéria seca em relação àquela contida inicialmente. Segundo Júnior et al. (2013) a aplicação de piraclostrobina causa incremento na matéria seca, conforme verificado pela taxa de crescimento relativo. Conforme Tsumanuma et al. (2010) a aplicação de estrobilurina associada a triazol em R2 e R5.1 em plantas de soja, resulta em maiores valores de taxas de crescimento absoluto, crescimento relativo e de crescimento da cultura.

Aárea foliar $\left(\mathrm{A}_{\mathrm{f}}\right)$ de plantas de tomateirocv. MicroTom ajustou-se ao modelo polinomial de terceiro grau (Tabela 1), o qual se caracteriza inicialmente com incremento acentuado de $\mathrm{A}_{\mathrm{f}}$ a partir dos 14
DAT em plantas de todos os tratamentos (Figura 1D). A máxima $A_{f}$ foi atingida aproximadamente aos 63 DAT em plantas dos tratamentos submetidos a solução nutritiva completa sem aplicação de piraclostrobina $\left(128,69 \mathrm{~cm}^{2}\right.$ planta-1 $\left.^{-1}\right)$ e com aplicação de piraclostrobina $\left(87,89 \mathrm{~cm}^{2}\right.$ planta $\left.^{-1}\right)$ apresentando valores superiores em relação a plantas dos tratamentos com solução nutritiva $1 / 2$ força de nitrogênio sem piraclostrobina $\left(76,24 \mathrm{~cm}^{2}\right.$ planta $\left.^{-1}\right)$ e com aplicação de piraclostrobina $(67,16$ $\mathrm{cm}^{2}$ planta $^{-1}$ ) que atingiram a máxima $\mathrm{A}_{\mathrm{f}}$ aos $59 \mathrm{DAT}$ com posterior tendência ao decréscimo.

A taxa de produção de área foliar $\left(\mathrm{C}_{\mathrm{a}}\right)$ foi máxima no início do ciclo de cultivo, aos 14DAT emplantas de todos os tratamentos, com tendência ao decréscimo ao longo do desenvolvimento das plantas (Figura 1E). Plantas submetidas ao tratamento solução nutritiva completa sem piraclostrobina apresentaram superior $\mathrm{C}_{\mathrm{a}}$, evidenciando a maior velocidade de crescimento de folhas comparativamente àquelas dos demais tratamentos. Similarmente, a taxa de crescimento relativo de área foliar $\left(\mathrm{R}_{\mathrm{a}}\right)$ (Figura $1 \mathrm{~F}$ ) apresentou tendência ao decréscimo ao longo da ontogenia, sendo os maiores valores de $\mathrm{R}_{\mathrm{a}}$ atingidos por plantas do tratamento $\mathrm{T} 2$ e os menores valores de $\mathrm{R}_{\mathrm{a}}$ por plantas do tratamento T4. Estes resultados demonstram que plantas de T2 apresentaram maior eficiência em aumentar sua área foliar em relação àquela contida inicialmente. 
Figura 1: Matéria seca total (A), taxa de produção de matéria seca (B), taxa de crescimento relativo de massa seca (C), área foliar (D), taxa de produção de área foliar (E), taxa de crescimento relativo de área foliar (F) de plantas de tomateiro cv. Micro-Tom submetidas ao nitrogênio e piraclostrobina. T1 - solução nutritiva completa sem aplicação de piraclostrobina; T2 - solução nutritiva completa + aplicação piraclostrobina; T3 - solução nutritiva $1 / 2$ força de nitrogênio sem aplicação de piraclostrobina e T4, solução nutritiva $1 \frac{1}{2}$ força de nitrogênio + aplicação de piraclostrobina.
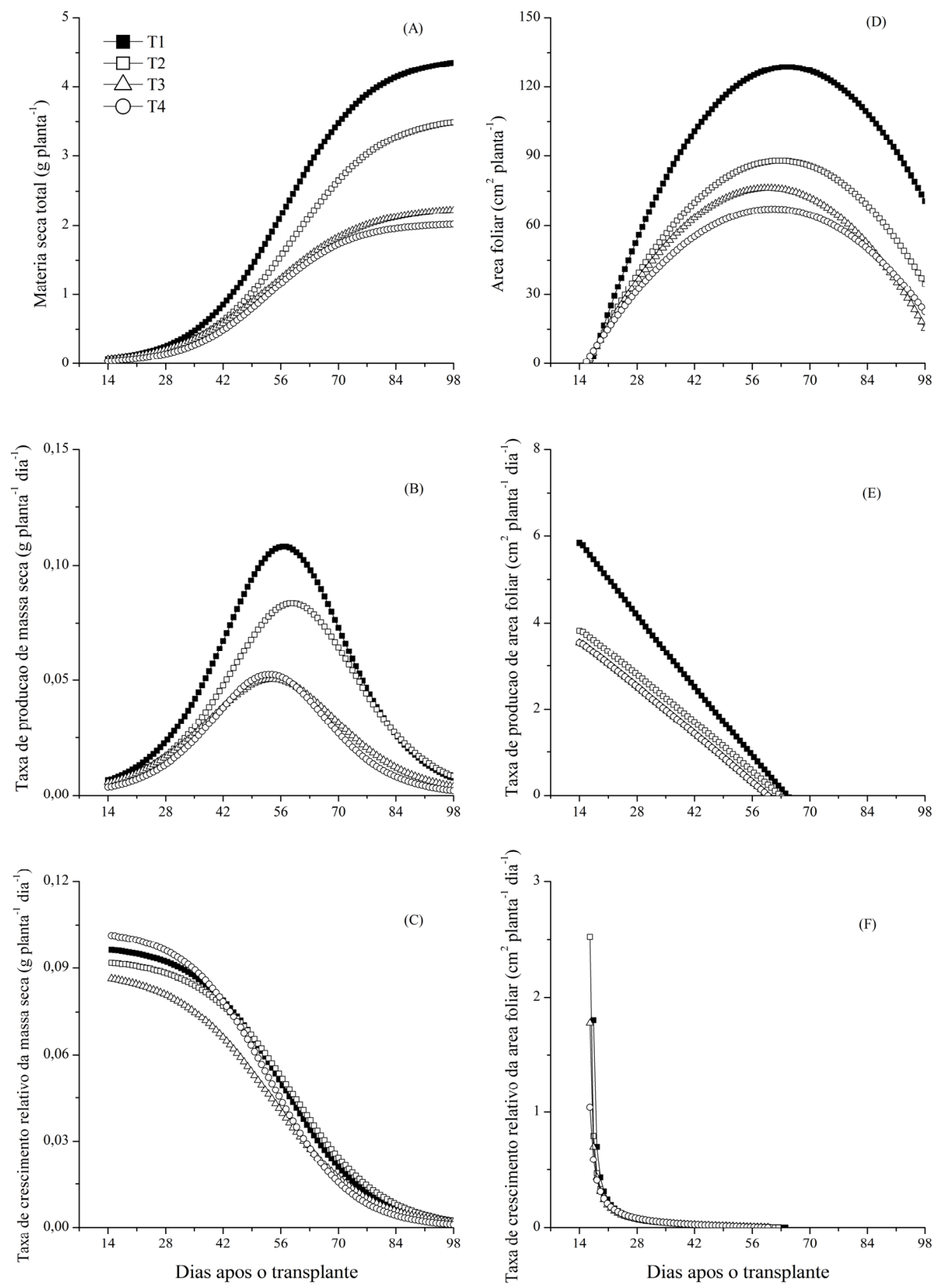

Fonte: Elaboração dos autores. 
A taxa assimilatória liquida $\left(\mathrm{E}_{\mathrm{a}}\right)$ foi máxima aos 21 DAT em plantas de todos os tratamentos, ocorrendo posterior declínio até 25 DAT aproximadamente em plantas submetidas ao tratamento com solução nutritiva $1 / 2$ força de nitrogênio + piraclostrobina (T4) e, até 28 DAT em plantas dos demais tratamentos (Figura 2A). No referido período, plantas submetidas ao tratamento $\mathrm{T} 4$ apresentaram menor $\mathrm{E}_{\mathrm{a}}$ comparativamente aos tratamentos T1, T2 e T3. Foi observado um segundo pico de $\mathrm{E}_{\mathrm{a}}$ aos $56,58,52$ e 52
DAT, nas plantas dos tratamentos T1, T2, T3 e T4, respectivamente. Neste sentido, mesmo as plantas de T2 tendo apresentado atraso temporal de 2 dias em relação às plantas de T1 e de 6 dias comparativamente àquelas de $\mathrm{T} 3$ ou $\mathrm{T} 4$, os valores atingidos foram superiores, evidenciando maior diferença entre a fotossíntese bruta e a respiração. Tal constitui indicativo de provável maior produção líquida de assimilados destinados à manutenção estrutural, ao crescimento e desenvolvimento da planta.

Figura 2. Taxa assimilatória líquida (A), razão de peso foliar (B), razão de área foliar (c) e área foliar específica (F) de plantas de tomateiro cv. Micro-Tom submetidas ao nitrogênio e piraclostrobina. T1 - solução nutritiva completa sem aplicação de piraclostrobina; T2 - solução nutritiva completa + aplicação piraclostrobina; T3 - solução nutritiva $1 / 2$ força de nitrogênio sem aplicação de piraclostrobina e T4, solução nutritiva $1 / 2$ força de nitrogênio + aplicação de piraclostrobina.
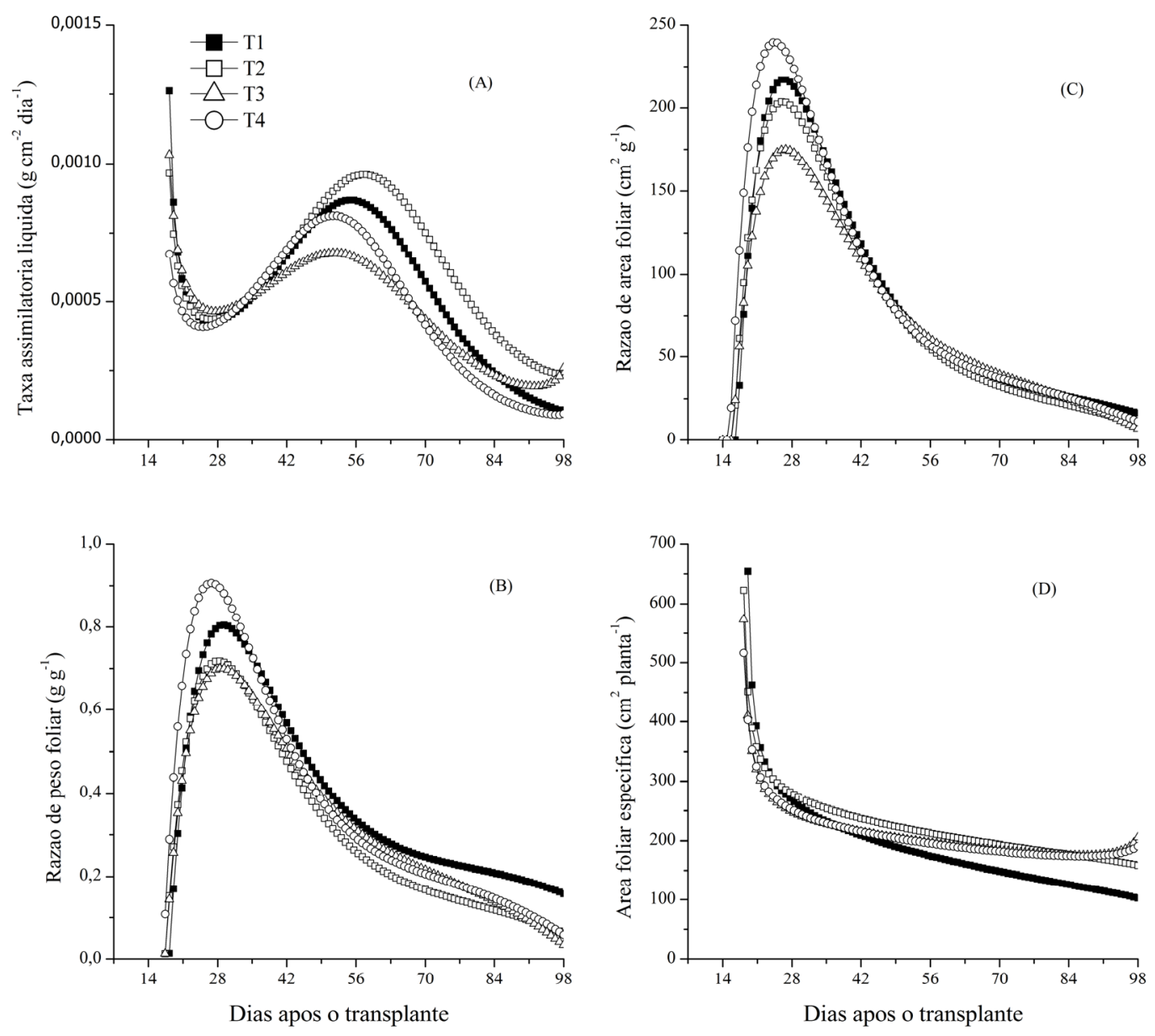

Fonte: Elaboração dos autores. 
A razão de massa foliar $\left(\mathrm{F}_{\mathrm{w}}\right)$, que expressa a relação entre as massas seca das folhas e total, foi máxima nas plantas do tratamento T4 aproximadamente aos 26 DAT seguidas por plantas submetidas a T1, T2 e T3 que atingiram o máximo $\mathrm{F}_{\mathrm{w}}$ aos $28 \mathrm{DAT}$, com posterior tendência a redução (Figura 2B). A maior $\mathrm{F}_{\mathrm{w}}$ no início do crescimento é devida, assim como $\mathrm{F}_{\mathrm{a}}$, a superior alocação de matéria seca nas folhas e constitui indicativo de que estes órgãos são dreno metabólico preferencial no início do ciclo de desenvolvimento da espécie. É possível afirmar que, em plantas submetidas a $\mathrm{T} 4$, as folhas constituíram-se em dreno metabólico mais forte, fato que pode manter relação ao menor número e massa de frutos comparativamente a plantas de T1 e T2 (Figura 3A e 3B).

Figura 3. Partição de assimilados de plantas de tomateiro cv. Micro-Tom submetidas ao nitrogênio e piraclostrobina. (A) solução nutritiva completa sem aplicação de piraclostrobina; (B) solução nutritiva completa + aplicação piraclostrobina; (C) solução nutritiva $1 \frac{1}{2}$ força de nitrogênio sem aplicação de piraclostrobina e (D) solução nutritiva $1 \frac{1}{2}$ força de nitrogênio + aplicação de piraclostrobina.

$$
\text { raiz } \Longrightarrow \text { caule } \square \text { folha } \because \because \text { flor } \mathrm{MW} \text { fruto }
$$

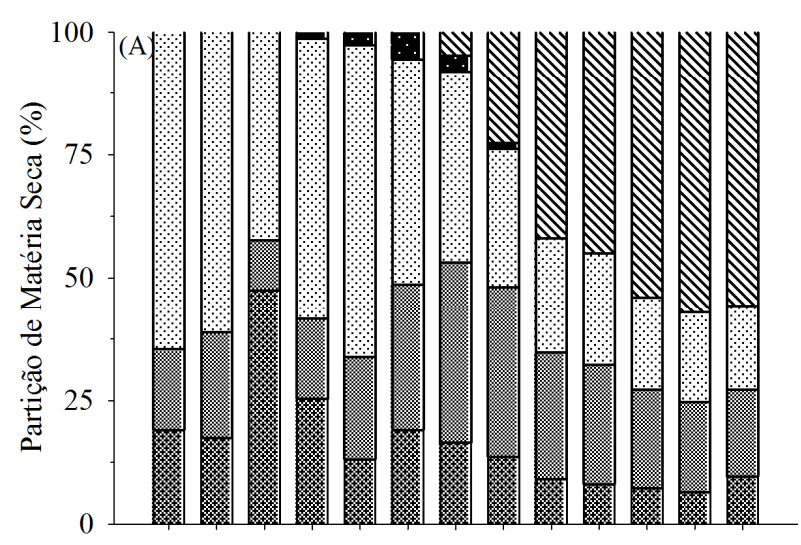

$\begin{array}{lllllllllllll}14 & 21 & 28 & 35 & 42 & 49 & 56 & 63 & 70 & 77 & 84 & 91 & 98\end{array}$

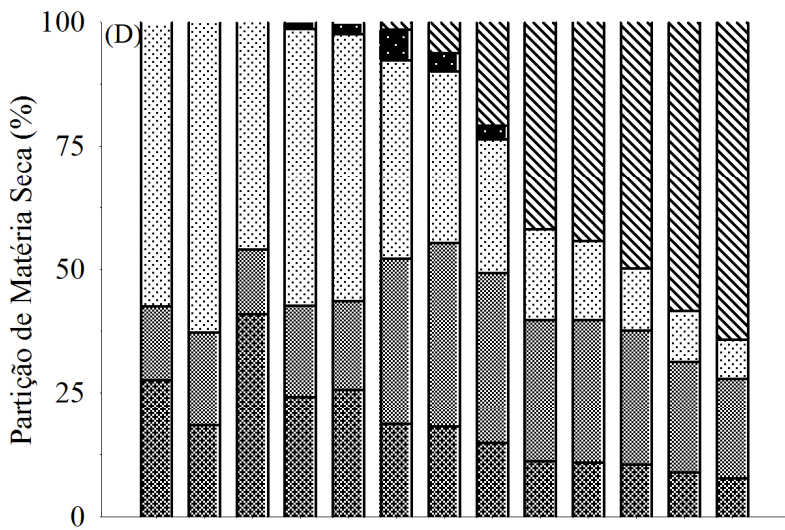

$\begin{array}{lllllllllllll}14 & 21 & 28 & 35 & 42 & 49 & 56 & 63 & 70 & 77 & 84 & 91 & 98\end{array}$

Dias após o transplante
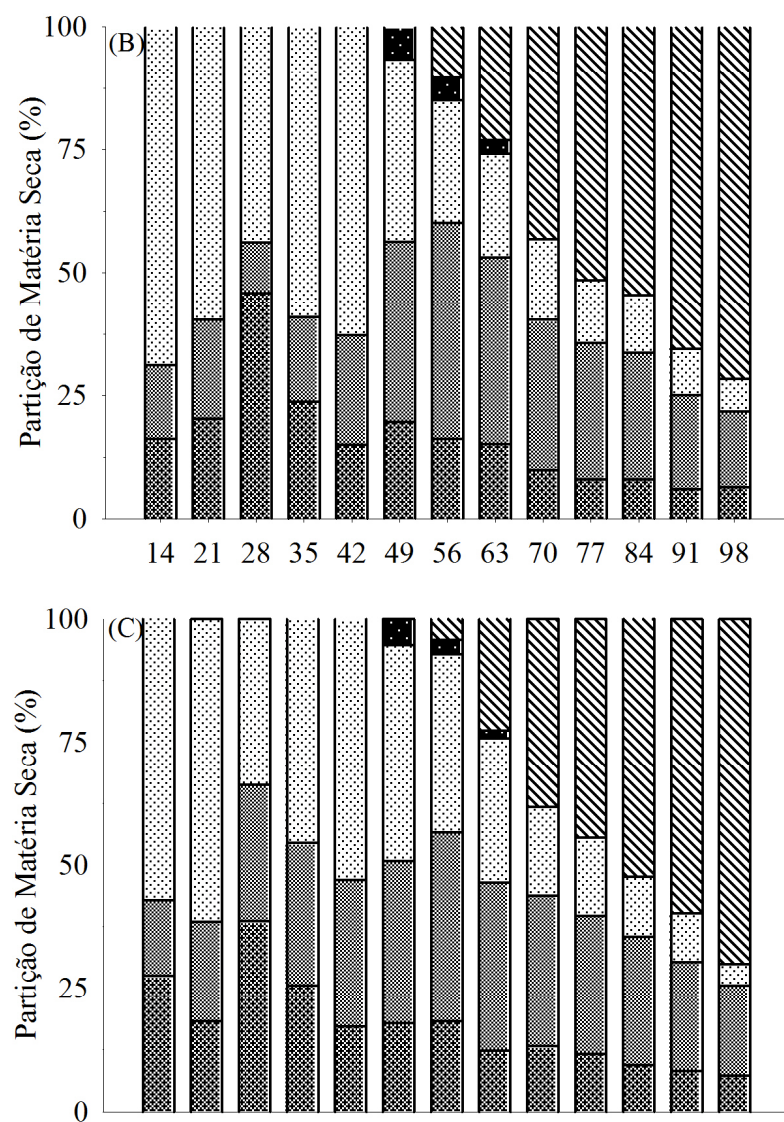

$\begin{array}{lllllllllllll}14 & 21 & 28 & 35 & 42 & 49 & 56 & 63 & 70 & 77 & 84 & 91 & 98\end{array}$

Dias após o transplante

Fonte: Elaboração dos autores. 
Os dados de razão de área foliar $\left(\mathrm{F}_{\mathrm{a}}\right)$, que expressa a relação entre a área e massa foliar, em plantas submetidas a todos os tratamentos, apresentaram tendência ao aumento acentuado no início do ciclo das plantas (Figura 2D), atingido valores máximos de: 216,97 $\mathrm{cm}^{-2} \mathrm{~g}^{-1}$ aos 26 DAT; 203,63 $\mathrm{cm}^{-2} \mathrm{~g}^{-1}$ aos 25 DAT; $175,07 \mathrm{~cm}^{-2} \mathrm{~g}^{-1}$ aos 26 DAT e $239,55 \mathrm{~cm}^{-}$ ${ }^{2} \mathrm{~g}^{-1}$ aos 24 DAT para as plantas dos tratamentos T1, $\mathrm{T} 2$, T3 e T4, respectivamente, posterior observou-se tendência ao decréscimo nos valores de $\mathrm{F}_{\mathrm{a}}$.

Os maiores valores de área foliar específica $\left(\mathrm{S}_{\mathrm{a}}\right)$ foram atingidos por plantas de todos os tratamentos aproximadamente aos 18 DAT com posterior tendência ao decréscimo até o final do ciclo de cultivo (Figura 2E). Cabe salientar que plantas não submetidas à associação nitrogênio e piraclostrobina apresentaram redução mais expressiva de $\mathrm{S}_{\mathrm{a}}$ a partir dos 56 DAT comparativamente as demais. É possível verificar que, indiferentemente ao tratamento, o período inicial caracterizou-se como de aumento na expansão foliar juntamente à redução da sua espessura, fato também observado após a metade final do ciclo das plantas submetidas à associação entre nitrogênio e piraclostrobina.

A partição de assimilados entre os diferentes órgãos das plantas de tomateiro cv. Micro-Tom foi modificada ao longo da ontogenia (Figura 3). O acúmulo de matéria seca nas raízes foi crescente até os 28 DAT para plantas de todos os tratamentos, sendo a quantidade máxima acumulada, em relação à matéria seca total, de 47, 46, 39 e 41\%, para as plantas dos tratamentos T1, T2, T3 e T4, respectivamente. Após o referido período, os tratamentos submetidos a solução nutritiva
$1 / 2$ força de nitrogênio, com ou sem aplicação de piraclostrobina (T3 e T4), mantiveram maior porcentagem de matéria seca quando comparados àqueles submetidos a solução nutritiva completa, com ou sem aplicação de piraclostrobina (T1 e T2).

A alocação de matéria seca em caule foi intensificada até os 56 DAT representando neste período $37,44,38$ e $37 \%$ da massa seca total das plantas dos tratamentos T1, T2, T3 e T4, respectivamente. Com o início da frutificação houve decréscimo na massa seca acumulada em tal órgão, em detrimento da mudança do dreno preferencial.

As folhas acumularam matéria seca até os 42 DAT em plantas dos tratamentos T1 (63.5\%), T2 (62.8\%) e T3 (53\%); e, até 35 DAT em plantas do tratamento T4 (56.2\%). Ao final do ciclo, os frutos representaram 56, 72, 70 e $64 \%$ da matéria seca total para plantas submetidas a T1, T2, T3 e T4, respectivamente.

Embora, a partição de assimilados entre os diferentes órgãos das plantas de tomateiro cv Micro-Tom tenha seguido padrão semelhante em plantas de todos os tratamentos, o início do período reprodutivo ocorreu aos 35 DAT em plantas de T1 e T4 e aos 49 DAT em plantas de T2 e T3 (Figura 3). O número de frutos $\left(\mathrm{N}_{\mathrm{fr}}\right)$ foi maior em plantas submetidas a solução nutritiva completa, com ou sem aplicação de piraclostrobina (T1 e T2) em relação àquelas submetidas a solução nutritiva $1 / 2$ força de nitrogênio, com ou sem aplicação de piraclostrobina (T3 e T4), que foram similares entre si (Tabela 2). Todavia, a massa média de frutos $\left(\mathrm{W}_{\mathrm{fr}}\right)$ foi reduzida pela associação nitrogênio e piraclostrobina.

Tabela 2: Número $\left(\mathrm{N}_{\mathrm{fr}}\right)$ e massa fresca de frutos em gramas $\left(\mathrm{W}_{\mathrm{fr}}\right)$ de plantas de tomateiro cv. Micro-Tom submetidas ao nitrogênio e piraclostrobina.

\begin{tabular}{cccc}
\hline Tratamentos & $\mathbf{N}_{\mathrm{fr}}$ & $\mathbf{W}_{\mathrm{fr}}$ \\
\hline T1 & $6,0 \mathrm{a}^{*}$ & 28,33 a \\
T2 & $6,3 \mathrm{a}^{\mathrm{a}}$ & $22,90 \mathrm{~b}$ \\
$\mathbf{T 3}$ & $3,8 \mathrm{~b}$ & $15,20 \mathrm{c}$ \\
$\mathbf{T 4}$ & $3,5 \mathrm{~b}$ & $12,63 \mathrm{c}$ \\
\hline
\end{tabular}

*Valores com a mesma letra na coluna não diferem significativamente entre si pelo teste de Tukey $(\leq 5 \%)$.

Fonte: Elaboração dos autores. 
Órgãos vegetativos devem se desenvolver o suficiente para interceptar luz e acumular água e nutrientes, contudo, o particionamento de carbono entre os vários grupos de órgãos depende do número de órgãos por grupo e de sua capacidade competitiva por fotoassimilados (VALANTIN et al., 1999). Por sua vez, a competição por fotoassimilados entre drenos ou entre dreno e fonte, afeta a taxa de crescimento da planta e a fixação dos frutos em muitas espécies. Assim, o aumento no número de frutos na planta pode aumentar a fração de fotoassimilados alocados nos frutos em detrimento das partes vegetativas (ANDRIOLO; FALCÃO, 2000), fato não observado no presente experimento.

No tomateiro, a alocação de fotoassimilados no dreno depende, principalmente, do número de frutos existentes na planta (BERTIN; GAUTIER; ROCHE, 2001). Plantas de meloeiro com apenas um fruto, comparadas a plantas com dois frutos alocaram, proporcionalmente, mais massa de matéria seca nas folhas e caule em detrimento dos frutos, levando a menor índice de colheita, apesar da maior massa média de frutos (QUEIROGA et al., 2008).

O investimento de assimilados e a remobilização do material fotossintético estão fortemente relacionados a fatores ambientais e a idade fisiológica do vegetal. Neste contexto, a disponibilidade de nutrientes é fator que influencia diretamente o referido processo, onde a deficiência de nitrogênio, decréscimos na assimilação de nitrato, na fotossíntese e na condutância estomática, são fatores que resultam em significantes reduções no crescimento das plantas (CARELLI et al., 1996).

O reduzido crescimento na fase inicial do desenvolvimento, é comum em diversas espécies vegetais e pode ser explicado pelo reduzido volume de raízes e pela baixa absorção de água e de minerais, pela pequena área foliar e pela reduzida taxa de respiração e taxa assimilatória líquida (AUMONDE et al., 2011). No início do ciclo, as folhas se comportaram como fonte-dreno, uma vez que são responsáveis pela produção de fotoassimilados e também o órgão com maior armazenamento de compostos (LOPES et al., 2011). Com o início da frutificação, ocorre $\mathrm{o}$ direcionamento dos assimilados das folhas para os frutos de forma mais intensa, já que tais órgãos passam a comportar-se como drenos preferenciais (LOPES et al., 2011), enquanto raízes, caules e folhas utilizam assimilados provenientes do processo fotossintético em menor intensidade com vistas a manutenção estrutural da planta.

Estudando o crescimento de plantas de sorgo sacarino cultivado em terrenos com diferentes exposições e declividades, Bacarin, Lopes e Benincasa (1997), relatam que a produção de matéria seca total mostrou três fases distintas, uma inicial anterior a floração, com crescimento relativamente lento, outra intermediária, do início da floração até o começo do período de enchimento da panícula, quando o crescimento foi acelerado e uma final, quando ocorreu o desenvolvimento das sementes e o acréscimo da matéria seca foi muito lento.

Em relação ao aumento na taxa de produção de matéria seca, que avalia o crescimento do vegetal relacionando a quantidade de material orgânico acumulado por unidade de tempo, pode estar associado, dentro de certos limites, a elevação da área foliar e taxa de produção de área foliar. Além disso, à quantidade de assimilados sintetizados e que se destinam ao crescimento e ao desenvolvimento vegetal (LOPES; MAESTRI, 1973). O aumento da área de folhas tem como objetivo elevar o potencial da fonte, inicialmente pela maior captação de energia luminosa para o processo fotossintético, visando à manutenção estrutural e o crescimento vegetal.

O decréscimo de $\mathrm{R}_{\mathrm{w}}$ ao longo do desenvolvimento, segundo Benincasa (1988), é resultado do aumento gradativo de tecidos não fotossintetizantes, devido à elevação da atividade respiratória, à variações em condições climáticas e ao autossombreamento que pode ser verificado pelo incremento da área foliar. Valores elevados de $\mathrm{R}_{\mathrm{w}}$ no início do ciclo devem-se ao fato da maior parte da área foliar da planta constituir-se de folhas jovens com elevada capacidade fotossintética e alta taxa de crescimento. 
Quanto a elevação de $\mathrm{E}_{\mathrm{a}}$, pode ser atribuída à maior demanda dos drenos por assimilados no período inicial de crescimento, visando a formação de área foliar para a fotossíntese e à diferenciação floral, formação e crescimento dos frutos durante a fase reprodutiva das plantas. $\mathrm{E}$, mesmo que $\mathrm{E}_{\mathrm{a}}$ tenha apresentando variabilidade temporal-quantitativa, é possível afirmar que as curvas apresentaram o padrão ontogênico esperado, isto porque, esta característica de crescimento não é determinada somente pela taxa fotossintética, mas também pela dimensão da área foliar, duração do período vegetativo, distribuição das folhas no dossel, ângulo foliar, translocação e partição de assimilados (AUMONDE et al., 2011).

Quando as plantas foram submetidas à associação $1 / 2$ dose de nitrogênio e a piraclostrobina houve indicativo de alteração nesta característica morfofisiológica de crescimento, ocorrendo aumento da área útil à fotossíntese em relação a plantas dos demais tratamentos. Entretanto, plantas de T4 mesmo com maior área foliar útil à fotossíntese, foram menos eficientes na produção líquida de assimilados (Figura 2A), fato que provavelmente, refletiu em menor produção de matéria seca total em comparação às demais plantas (Figura 1A).

No início do desenvolvimento, diversas espécies vegetais apresentam elevação de $F_{a}$, o que se deve ao maior investimento de assimilados na formação de folhas visando maior captação de radiação solar (URCHEI; RODRIGUES; STONE, 2000). A tendência de redução de $\mathrm{F}_{\mathrm{a}}$ ao longo do ciclo da espécie, é explicada em parte, pelo aumento gradual de tecidos não assimilatórios e partes reprodutivas que se constituem em dreno preferencial forte e definitivo (Figura 3). Por outro lado, a redução de $\mathrm{F}_{\mathrm{w}}$ é comum, pois ao longo do desenvolvimento e com a formação dos órgãos reprodutivos, as folhas deixam de ser dreno metabólico preferencial e exportam seus assimilados para os referidos órgãos (LOPES; MAESTRI, 1973).

A partir de uma análise conjunta e comparativa dos dados de crescimento e partição de assimilados de plantas de tomateiro cv. Micro-Tom é possível verificar que plantas submetidas a T1 apresentaram maior $\mathrm{W}_{\mathrm{t}}, \mathrm{C}_{\mathrm{t}}$ e $\mathrm{W}_{\mathrm{fr}}$ em relação àquelas dos demais tratamentos. Entretanto, plantas de T2 apresentaram maior e similar $\mathrm{E}_{\mathrm{a}}, \mathrm{N}_{\mathrm{fr}}$ a plantas de $\mathrm{T} 1$, alocando em relação à matéria seca total e ao final do ciclo, maior porcentagem de matéria seca nos frutos. Desse modo, é possível inferir que a associação entre nitrogênio e piraclostrobina altera características de crescimento e de partição de assimilados em plantas da referida espécie, influenciando em características produtivas das plantas.

\section{Conclusões}

A associação concentração de nitrogênio e piraclostrobina altera o crescimento e a partição de assimilados em plantas de tomateiro cv. Micro-Tom.

Plantas submetidas à $1 / 2$ dose de nitrogênio apresentam maior massa seca total, similar número de frutos e menor porcentagem final de matéria seca total em frutos, comparativamente, àquelas submetidas à associação $1 / 2$ dose de nitrogênio e a piraclostrobina.

\section{Agradecimentos}

Coordenação de Aperfeiçoamento de Pessoal de Nível Superior (CAPES) e Conselho Nacional Pesquisa (CNPq).

\section{Referências}

ANDRIOLO, J. L.; FALCÃO, L. L. Efeito da poda de folhas sobre a acumulação de matéria seca e sua repartição para os frutos do tomateiro cultivado em ambiente protegido. Revista Brasileira de Agrometeorologia, Sete Lagoas, v. 8, n. 1, p. 75-83, 2000.

AUMONDE, T. Z.; LOPES, N. F.; MORAES, D. M.; PEIL, R. M. N.; PEDÓ, T. Análise de crescimento do híbrido de mini melancia Smile $^{\circledR}$ enxertada e não enxertada. Interciência, Caracas, v. 36, n. 9, p. 677-681, 2011.

BACARIN, M. A.; LOPES, N. F.; BENINCASA, M. Crescimento de sorgo sacarino, Sorghum Bicolor (L.) Moench, cultivado em terrenos com diferentes exposições e declividades. 1. Análise de crescimento. Ciência e Agrotecnologia, Lavras, v. 21, p. 286-293, 1997. 
BARTLETT, D. W.; CLOUGH, J. M.; GODWIN, J. R.; HALL, A. A.; HAMER, M.; PARR-DOBRZANSKI, B. The strobilurin fungicides. Pest Management Science, Sussex, v. 58, n. 7, p. 649-662, 2002.

BENINCASA, M. M. P. Análise de crescimento de plantas: noções básicas. Jaboticabal: Fundação de Estudos e Pesquisas em Agronomia, Medicina Veterinária e Zootecnia, 1998. 41 p.

BERTIN, N.; GAUTIER, H.; ROCHE, C. Number of cells in tomato fruit depending on fruit position and source-sink balance during plant development. Plant Growth Regulation, New York, v. 36, n. 2, p. 105-112, 2001.

BRASIL. Ministério da Agricultura e Reforma Agrária. Regras para análise de sementes. Brasília: SNAD/ CLAV, 2009. 398 p.

CARELLI, M. L. C.; UNGARO, M. R. G.; FAHL, J. I.; NOVO, M. C. S. S. Níveis de nitrogênio, metabolismo, Crescimento e produção de girassol. Revista Brasileira de Fisiologia Vegetal, Londrina, v. 8, n. 2, p. 123-130, 1996.

CASTRO, P. R. C.; PITELLI, A. M. C. M.; PERES, L. E. P.; ARAMAKI, P. H. Análise da atividade reguladora de crescimento vegetal de tiametoxam através de biotestes. Publicatio, Ponta Grossa, v. 13, n. 3, p. 25-29, 2007.

GROSSMANN, K.; RETZLAFF, G. Bioregulatory effects of the fungicidal strobilurin kresoxim-methyl in wheat (Triticum aestivum). Pesticide Science, Oxford, v. 50, n. 1, p. 11-20, 1997.

HOAGLAND, D. R.; ARNON, D. I. The water-culture method for growing plants without soil. California Agricultural Experiment Station Circular, Berkeley, v. 347, p. 1-32, 1950.

JÚNIOR, J. P.; FAGAN, E. B.; CORRÊA, L. T.; SOARES, J. N.; PEREIRA, I. S.; SILVA, L. G. Resposta fisiológica de mudas de café à aplicação foliar de estrobilurinapiraclostrobina e silício. Cerrado Agrociencias, Patos de Minas, v. 4, p. 42-57, 2013.

KÖHLE, H.; GROSSMANN, H.; JABS, T.; GERHARD, M.; KAISER, W.; GLAAB, J.; CONRATH, U.; SEEHAUS, K.; HERMS, S. Physiological effects os the strobilurin fungicide F500 on plants. In: DEHNE, H, W.; GISI, U.; KUCK, K. H.; RUSSELL, P. E.; LYR, H. (Ed.). Modern Fungicides and Antigungal Compounds III. Andover: Intercept, 2002. p. 61-74.

LOPES, N. F.; MAESTRI, M. Análise de crescimento e conversão de energia solar em milho (Zea mays L.) em Viçosa, Minas Gerais. Ceres, Viçosa, v. 20, n. 109, p. 189-201, 1973.
LOPES, W. A. R.; NEGREIROS, M. Z.; DOMBROSKI, J. L. D.; RODRIGUES, G. S. O.; SOARES, A. M.; ARAÚJO, A. P. Análise do crescimento de tomate 'SM-16' cultivado sob diferentes coberturas de solo. Horticultura Brasileira. Brasilia, v. 29, n. 4, p. 554-561, 2011.

MARTÍ, E.; GISBERT, C.; BISHOP, G. J.; DIXON, M. S. Genetic and physiological characterization of tomato cv. Micro-Tom. Journal of Experimental Botany, Oxford, v. 57, n. 9, p. 2037-2047, 2006.

QUEIROGA, R. C. F.; PUIATTI, M.; FONTES, P. C. R.; CECON, P. R. Partição de assimilados e índices fisiológicos de cultivares de melão do grupo Cantalupensis influenciados por número e posição de frutos na planta, em ambiente protegido. Revista Ceres, Viçosa, v. 55, n. 6, p. 596-604, 2008.

RADFORD, P. J. Growth analysis formulae: their use and abuse. Crop Science, Madison, v. 7, n. 3, p. 171-175, 1967.

RICHARDS, F. J. The quantitative analysis of growth. In: STEWWARD, F. C. (Ed.). Plant physiology. New York: Academic Press, 1969. p. 3-76.

TAIZ, L.; ZEIGER, E. Fisiologia vegetal. Porto Alegre: Artmed, 2013. $820 \mathrm{p}$.

TSUMANUMA, G. M.; PINTO DE CARVALHO, S. J.; FANCELLI, A. L.; SILVEIRA BERNARDES, M.; TAVARES RODRIGUES, M. A.; BEGLIOMINI, E. Crescimento de dois cultivares de soja submetidos a aplicações de herbicidas e fungicidas. Revista Ceres, Viçosa, v. 57, n. 6, p. 742-750, 2010.

URCHEI, M. A.; RODRIGUES, J. D.; STONE, L. F. Análise de crescimento de duas cultivares de feijoeiro sob irrigação, em plantio direto e preparo convencional. Pesquisa Agropecuária Brasileira, Rio de Janeiro, v. 35, n. 3, p. 497-506, 2000.

VALANTIN, M.; GARY, C.; VAISSIÈRE, B. E.; FROSSARD, J. S. Effect of fruit load on partitioning of dry matter and energy in cantaloupe (Cucumis melo L.). Annals of Botany, London, v. 84, n. 2, p. 173-181, 1999.

VENANCIO, W. S.; RODRIGUES, M. A. T.; BEGLIOMINI, E.; SOUZA, N. L. D. Physiological effects of strobilurin fungicides on plants. Publicatio UEPG: Ciências Exatas e da Terra, Ciências Agrárias e Engenharias, Ponta Grossa, v. 9, n. 3, p. 59-68, 2003.

ZHANG, Y. J.; ZHANG, X.; CHEN, C. J.; ZHOU, M. G.; WANG, H. C. Effects of fungicides JS399-19, azoxystrobin, tebuconazloe, and carbendazim on the physiological and biochemical indices and grain yield of winter wheat. Pesticide Biochemistry and Physiology, San Diego, v. 98, n. 2, p. 151-157, 2010. 